\title{
AUTHOR INDEX VOLUME 17
}

Abd-Rahman MK, see Dimyati K

$17(2008) 193$

Abdul-Rashid HA, see Harun $S W$

17 (2008) 199

Abobaker AM, Moubissi AB, Ekogo ThB \& Nakkeeran K, Analytical Solutions for the Variational Equations Derived for the Nonlinear Schrödinger Equation: Dispersion-Managed Fiber System

Abu Hassan H, see Chuah LS

Ahmad H, see Dimyati $K$

Ahmad H, see Harun $S W$

Ailing T, see Hongjun $W$

Akinaga H, see Sinsarp A

Alatas H, Kandi AA, Iskandar AA \& Tjia MO, New Class of Bright Spatial Solitons Obtained by Hirota's Method from Generalized Coupled Mode Equations of Nonlinear Optical Bragg Grating

Ali J, see Saktioto

Aly AH, Ryu S-W \& Wu C-J, Electromagnetic Wave Propagation Characteristics in a One-Dimensional Metallic Photonic Crystal

Bartwal KS, see Sen P

Batarfi HA, Dispersive Switching in Bistable Models

Bingcai L, see Hongjun $W$

Blair J, see Summers CJ

Blinov LM, Chidichimo G, Cipparrone G, Pagliusi P \& Rugiero T, Band Narrowing and Gain Spectra of Laser Dye Solutions with Scattering $\mathrm{TiO}_{2}$ Nanoparticles

Boulezhar A, see Brunel $M$

Brunel M, Mgharaz D, Coetmellec S \& Boulezhar A, Non-Diverging Dark-Hollow Beam Generation with a Pigtailed Laser Diode

Brunsen A, see Knoll W

Cao GR, see Yang DY

Castro E, see Mendoza-García A

Chakrabarti S \& Ramakrishna SA, Design of Metallic Metamaterial Structures at High Frequencies

17 (2008) 285

$17(2008) 59$

17 (2008) 193

17 (2008) 199

17 (2008) 503

17 (2008) 105

17 (2008) 225

17 (2008) 111

17 (2008) 255

17 (2008) 175

17 (2008) 265

$17(2008) 503$

17 (2008) 1

17 (2008) 71

17 (2008) 305

17 (2008) 305

17 (2008) 121

17 (2008) 443

17 (2008) 511

17 (2008) 143

17 (2008) 315

17 (2008) 71

17 (2008) 435

17 (2008) 59

17 (2008) 175 
Chuah LS, Hassan Z \& Hassan HA, Electrical Resistance of Crack-Free GaN/AlN Heterostructure Grown on Si(111)

17 (2008) 299

Chuah LS, Hassan Z, Abu Hassan H, Chin CW \& Thahab SM, Large Area GaN Metal Semiconductor Metal (MSM) Photodiode Using a Thin Low Temperature GaN Cap Layer

Cipparrone G, see Blinov LM

Coetmellec S, see Brunel $M$

Cui Y, see Wang $X$

Cui Y, see Zhu $X$

Cui Z, see Shi $Z$

Danilov EO, see $H u Y$

Das M, Trivedi R, Khattak BQ, Ramshankar P, Sen P, Dolui SK \& Sen PK, Second Harmonic Generation in $\Lambda$-Shaped Organic Molecules

Das M, Trivedi R, Sen PK, Khattak BQ, Shankar PR, Prasad R \& Sen P, Geometry Optimization and Second Harmonic Generation in Para-Nitroaniline-Dimethyl Formamide Adduct

Dimyati K, Harun SW, Muhd-Yassin SZ, Abd-Rahman MK \& Ahmad H, Compact and Efficient Er-Yb-Doped Fiber Amplifier

Dolui SK, see Das $M$

Dostálek J, see Knoll W

$\mathrm{Du}$ C, see Ruan $S$

Ekogo ThB, see Abobaker AM

Fadhali M, see Saktioto

Ferrara MA, see Sirleto L

Ferreira MFS, Nonlinear Effects in Optical Fibers: Limitations and Possibilities

Fiddy MA, Ingel RP \& Schenk JO, Anisotropic Metamaterials for Field Enhancement and Negative Index Applications

Friberg AT, see Zhang $R$

Fukushima H, see Keum $C$-D

Furuhashi H, see Wang $X$

Furusawa M, see Keum $C$-D

Gaillot DP, see Summers CJ

Gorayeb M, see Mastrodomenico A

Gorayeb M, see Mendoza-García A

Graugnard E, see Summers CJ

Hara M, see Wulandari $P$

$17(2008) 59$

17 (2008) 71

17 (2008) 305

17 (2008) 451

17 (2008) 473

17 (2008) 243

17 (2008) 275

17 (2008) 329

$17(2008) 85$

17 (2008) 193

17 (2008) 329

17 (2008) 121

17 (2008) 425

17 (2008) 285

17 (2008) 111

17 (2008) 37

17 (2008) 23

17 (2008) 357

17 (2008) 367

17 (2008) 131

17 (2008) 451

17 (2008) 131

17 (2008) 1

17 (2008) 213

17 (2008) 511

17 (2008) 1

17 (2008) 185

Harun SW, Shirazi MR, Abdul-Rashid HA \& Ahmad H, Multiwavelength Source Using a Brillouin Fiber Laser

17 (2008) 199

17 (2008) 193

Harun SW, see Dimyati K

Hassan HA, see Chuah LS

17 (2008) 299

Hassan SS, see Sharaby YA

17 (2008) 339

Hassan Z, see Chin $C W$

17 (2008) 435

Hassan Z, see Chuah LS

17 (2008) 59

17 (2008) 299

Hassan Z, see Chuah LS

17 (2008) 413

Haus JW, see $W u Z$

17 (2008) 349

Hernández AJ, see Mendoza-García A

17 (2008) 511 
Hongjun W, Zhao W, Ailing T \& Bingcai L, Modeling and Analysis of Commonpath Phase Shifting Shear System Based on Birefringent Optical Devices

$17(2008) 503$

$\mathrm{Hu}$ Y, Danilov EO, Wex B \& Neckers DC, Relationship of Molecular Structure to Nonlinear Optical Properties of Organic Heterocyclic Compounds

Huang L-Y, see Jiang $Y-Y$

Huang W, see Zhu $X$

Huang Y, see Ruan $S$

Ingel RP, see Fiddy $M A$

Ishii $\mathrm{N}$, see Keum $C$ - $D$

Iskandar AA, see Alatas $H$

Jalali B, see Sirleto $L$

Ji K, Nasu K, Namikawa K \& Zheng H, Dynamics of Ferroelectric Nano Cluster in $\mathrm{BaTiO}_{3}$ Observed as a Real Time Correlation Between Two Soft X-Ray Laser Pulses

Jiang Y-Y \& Shen Z-J, Guiding Characteristics of an Air Waveguide with Left-Handed Metamaterials Cladding

Jiang Y-Y, Li J \& Huang L-Y, Beam Coupling Characteristics in Nematic Liquid Crystal Doped with Silver Nanoparticles

Jin Y \& He S, Impedance-Matched Multilayered Structure Containing a Zero-Permittivity Material for Spatial Filtering

Kandi AA, see Alatas $H$

Kar S, see Sen $P$

Karjanto N, see Susanto $H$

Kasry A, see Knoll W

Keum C-D, Ishii N, Michioka K, Wulandari P, Tamada K, Furusawa M \& Fukushima H, A Gram Scale Synthesis of Monodispersed Silver Nanoparticles Capped by Carboxylates and Their Ligand Exchange

Khang NC \& van Minh N, Synthesis of Anatase $\mathrm{TiO}_{2}$ Particles and Their Size-Related Structural, Optical and Raman Spectroscopy Studies

Khattak BQ, see Das $M$

Khattak BQ, see Das $M$

Khoi NT, see Thuy NM

Khunsin W, Romanov SG \& Torres CMS, Rotational Symmetry of Transmission Patterns and Ordering of Opal Photonic Crystals

Knoll W, Kasry A, Yu F, Wang Y, Brunsen A \& Dostálek J, New Concepts with Surface Plasmons and Nano-Biointerfaces

Li J, see Jiang $Y$ - $Y$

Li X, see Sun $X$

Li X, see Wulandari $P$

Li X, see $X u L$

Liu J, see Wang $X$

Liu W-J, Tian B \& Xu T, Symbolic Computation Study of Bright Solitonic Pulses in the Normal Dispersion Region

Lu C, see Zhu $X$

Lui LFK, see Nakkeeran $K$

Maity GK, see Chattopadhyay $T$

Manago T, see Sinsarp A

17 (2008) 275

17 (2008) 377

17 (2008) 473

17 (2008) 425

17 (2008) 357

17 (2008) 131

17 (2008) 225

17 (2008) 37

$17(2008) 395$

17 (2008) 465

$17(2008) 377$

17 (2008) 349

17 (2008) 225

17 (2008) 175

17 (2008) 159

17 (2008) 121

17 (2008) 131

17 (2008) 167

17 (2008) 85

17 (2008) 329

17 (2008) 205

17 (2008) 97

17 (2008) 121

17 (2008) 377

17 (2008) 387

17 (2008) 185

17 (2008) 405

17 (2008) 451

17 (2008) 235

17 (2008) 473

17 (2008) 15

17 (2008) 315

17 (2008) 105 
Martín P, see Mendoza-García A

$17(2008) 511$

Mastrodomenico A, Gorayeb M \& Paz JL, Collisional Effect of the Solvent on the Optical Responses of a Two-Level System

Mendoza-García A, Paz JL, Gorayeb M, Hernández AJ, Castro E \& $17(2008) 213$ Martín P, Solvent Effects in the Determination of the Nonlinear Optical Properties

Mgharaz D, see Brunel $M$

Michioka K, see Keum $C$-D

Mizutani T, see Wang $X$

Moubissi AB, see Abobaker AM

Muhd-Yassin SZ, see Dimyati $K$

Nakkeeran K, Wai PKA, Xu L, Lui LFK \& Tam HY, Role of Hysteresis on the Mode-Shift Characteristics of Injection Locking a Laser Diode

Nakkeeran K, see Abobaker AM

Namikawa K, see Ji K

Nasu K, see Ji K

Neckers DC, see $H u Y$

Neff CW, see Summers CJ

Nelson RL, see $W u Z$

Ochiai S, see Wang $X$

Ouyang Q, Zhang X \& Song Y, The Third- and Fifth-Order Nonlinear Refraction in the Single- and Double-Sided TMAF/PSS Electrostatic Self-Assembly Films

Pagliusi P, see Blinov LM

Paz JL, see Mastrodomenico A

Paz JL, see Mendoza-García A

Pei Y, see Yao F

Popov S, see Zhang $R$

Prasad R, see Das $M$

Qian Y, see Zhu X

Qiu FX, see Yang DY

Ramakrishna SA, see Chakrabarti $S$

Ramshankar P, see Das $M$

Rendina I, see Sirleto $L$

Ricciardi S, see Zhang $R$

Romanov SG, see Khunsin W

Roy JN, see Chattopadhyay $T$

$\mathrm{Ru}$ J, see Wang $X$

Ruan S, Huang Y, Du C \& Yu Y, Laser Direct Writing of Long Period Fiber Grating by 800 nm Femtosecond Laser Pulses

Rugiero T, see Blinov LM

Ryu S-W, see Aly AH

Saktioto, Ali J, Fadhali M \& Zainal J, Estimation of the Coupling Power Parameter of Fused Coupled Fibers

Sam DT, see Thuy NM

Schenk JO, see Fiddy MA

Sen P, Sisodia N, Choubey RK, Kar S \& Bartwal KS, Effect of $M g O$ Doping on Coercive Field in $\mathrm{LiNbo}_{3}$ Crystals

Sen $\mathrm{P}$, see Das $M$

$17(2008) 511$

17 (2008) 305

17 (2008) 131

17 (2008) 451

17 (2008) 285

17 (2008) 193

$17(2008) 15$

17 (2008) 285

$17(2008) 395$

17 (2008) 395

17 (2008) 275

17 (2008) 1

17 (2008) 413

17 (2008) 451

17 (2008) 487

17 (2008) 71

17 (2008) 213

17 (2008) 511

17 (2008) 495

17 (2008) 367

17 (2008) 85

17 (2008) 473

17 (2008) 443

17 (2008) 143

17 (2008) 329

17 (2008) 37

17 (2008) 367

17 (2008) 97

17 (2008) 315

17 (2008) 451

17 (2008) 425

17 (2008) 71

17 (2008) 255

17 (2008) 111

17 (2008) 205

17 (2008) 357

17 (2008) 175

17 (2008) 85 
Sen $\mathrm{P}$, see Das $M$

Sen PK, see Das $M$

Sen PK, see Das $M$

Sergeyev S, see Zhang $R$

Shankar PR, see Das $M$

Sharaby YA \& Hassan SS, Dispersive Switching in Mesoscopic Multistable Systems

17 (2008) 329

17 (2008) 85

17 (2008) 329

17 (2008) 367

17 (2008) 85

17 (2008) 339

17 (2008) 465

Shen Z-J, see Jiang $Y$ - $Y$

Shi Z, Zhang X \& Cui Z, Synthesis and Characterization of Thiophene-Containing Chromophores for Nonlinear Optical (NLO) Materials

Shirazi MR, see Harun $S W$

Sinsarp A, Manago T, Takano F \& Akinaga H, Electrical Spin Injection from an Iron-Rich Iron-Platinum Thin Film into Gallium Arsenide

17 (2008) 243

17 (2008) 199

$17(2008) 105$

Sirleto L, Ferrara MA, Rendina I \& Jalali B, Raman Approach in Porous Silicon at $1.5 \mu M$

Sisodia N, see Sen $P$

Song Y, see Ouyang $Q$

Summers CJ, Graugnard E, Gaillot DP, Yamashita T, Neff CW \& Blair J, Tuning of Photonic Crystal Band Properties by Atomic Layer Deposition

Sun X, Li X \& Zhang J, Orientating Manipulation of Cylindrical Particles with Optical Tweezers

Sun X, see Yao F

Susanto H \& Karjanto N, Calculated Threshold of Supratransmission Phenomena in Waveguide Arrays with Saturable Nonlinearity

Takano F, see Sinsarp A

Tam HY, see Nakkeeran $K$

Tamada K, see Keum C-D

Tamada K, see Wulandari $P$

Thahab SM, see Chuah LS

Thi TM, see Thuy NM

Thuy NM, Sam DT, Thi TM \& Khoi NT, Synthesis of Mn-doped ZnS and the Dopant-Induced Photoluminescence Property

Tian B, see Liu W-J

Tjia MO, see Alatas $H$

Torres CMS, see Khunsin W

Trivedi R, see Das $M$

Trivedi R, see Das $M$

Uchida Y, see Wang $X$

van Minh $\mathrm{N}$, see Khang $N C$

Wai PKA, see Nakkeeran $K$

Wang X, Ru J, Ochiai S, Yamada Y, Uchida Y, Furuhashi H,

Mizutani T, Cui Y \& Liu J, Thickness Dependence of

Third-Harmonic Generation from Self-Assembled Regioregular

Poly(3-Hexylthiophene) Thin Films on Quartz Glasses with

Different Surfaces

17 (2008) 451

Wang Y, see Knoll W

17 (2008) 121

Wex B, see $H u Y$

17 (2008) 275

$17(2008) 1$

17 (2008) 387

17 (2008) 495

17 (2008) 159

17 (2008) 105

17 (2008) 15

17 (2008) 131

17 (2008) 185

17 (2008) 59

17 (2008) 205

17 (2008) 205

17 (2008) 235

17 (2008) 225

17 (2008) 97

17 (2008) 85

17 (2008) 329

17 (2008) 451

17 (2008) 167

17 (2008) 15 
Wu C-J, see Aly AH

17 (2008) 255

Wu Z, Haus JW, Zhan Q \& Nelson RL, Long Range Surface Plasmon Devices Design Using Subwavelength Metal Grating

17 (2008) 413

Wulandari P, Li X, Tamada K \& Hara M, Conformational Study of Citrates Adsorbed on Gold Nanoparticles Using Fourier Transform Infrared Spectroscopy

Wulandari P, see Keum, C-D

$\mathrm{Xu}$ L, Li X \& Yuan J, Effect of Substrate Temperature on the Microstructure and Optical Properties of ZnO Nanocrystalline Thin Films

Xu L, see Nakkeeran $K$

$\mathrm{Xu} \mathrm{T}$, see Liu $W-J$

Yam FK, see Chin $C W$

Yamada $\mathrm{Y}$, see Wang $X$

Yamashita T, see Summers CJ

Yang DY, Qiu FX \& Cao GR, Synthesis and Electro-Optic Property of Intercalation Polyimide and Nanohybrid

$17(2008) 131$

17 (2008) 405

17 (2008) 15

17 (2008) 235

17 (2008) 435

17 (2008) 451

17 (2008) 1

$17(2008) 443$

Yang P-K, Determining the Third-Harmonic Susceptibility by Fourier Analysis

Yao F, Pei Y \& Sun X, Current Rectification in Nematic Liquid Crystal Cell with Alkanethiol Self-Assembled Monolayer

Yu F, see Knoll W

Yu Y, see Ruan $S$

Yuan J, see $X u L$

Zainal J, see Saktioto

Zhan $\mathrm{Q}$, see $W u Z$

Zhang J, see Sun $X$

Zhang R, Popov S, Ricciardi S, Friberg AT \& Sergeyev S, Distortion Tolerance Against Geometry Imperfections in Polymeric Microcavity Dye Laser

Zhang X, see Ouyang $Q$

Zhang X, see Shi Z

Zhao W, see Hongjun $W$

Zheng $\mathrm{H}$, see Ji $K$

Zhu X, Qian Y, Huang W, Lu C \& Cui Y, Synthesis, Fluorescence and Electrochemical Properties of Symmetrical Chromophores with Electron Accepting Oxadiazole

17 (2008) 49

17 (2008) 495

17 (2008) 121

17 (2008) 425

17 (2008) 405

17 (2008) 111

17 (2008) 413

17 (2008) 387

17 (2008) 367

17 (2008) 487

17 (2008) 243

17 (2008) 503

17 (2008) 395

17 (2008) 473 